\section{THU0411 CLINICAL, ECONOMIC, AND HUMANISTIC BURDEN ASSOCIATED WITH DELAYED DIAGNOSIS OF AXIAL SPONDYLOARTHRITIS: A SYSTEMATIC LITERATURE REVIEW}

Esther $\mathrm{Yi}^{1}{ }^{1,2}$, Amit Ahuja ${ }^{3}$, Tanvi Rajput ${ }^{3}$, Aneesh George ${ }^{3}$, Yujin Park ${ }^{4} .{ }^{1}$ The University of Texas at Austin, Austin, United States of America; ${ }^{2}$ Baylor Scott and White Health, Temple, United States of America; ${ }^{3}$ Novartis Healthcare Pvt Ltd, Hyderabad, India; ${ }^{4}$ Novartis Pharmaceuticals Corporation, East Hanover, United States of America

Background: Delayed diagnosis in patients with axial spondyloarthritis (AxSpA) has been shown to negatively impact disease prognosis and contributes to worse economic and quality of life outcomes; however, there is limited evidence available regarding the association of delayed diagnosis of AxSpA with the comprehensive burden of disease.

Objectives: To identify and summarize current published literature evaluating the clinical, economic, and humanistic burden associated with delayed diagnosis in patients with AxSpA.

Methods: This systematic literature review was conducted and reported according to the PRISMA guidelines (Figure 1. .). ${ }^{1}$ Publications were retrieved from the MEDLINE ${ }^{\circledast}$ (including MEDLINE ${ }^{\oplus}$ In-Process) and Embase $^{\circledast}$ databases. English-language publications of original research articles (up to July 12, 2018) and conference abstracts (2014 to 2018) reporting studies of delayed diagnosis of adult patients with AxSpA associated with clinical, economic, or humanistic burden were eligible for inclusion. Abstracts from all records retrieved from the literature search were screened for eligibility by two independent reviewers (first-level screening); discrepancies between reviewers were resolved by a third independent reviewer. Citations that did not match the eligibility criteria and duplicates of citations were excluded at the abstract screening stage. Full-text publications underwent second-level screening as described for first-level screening. Data were extracted from all records that met the eligibility criteria after second-level screening.

Results: Of the 1391 publications retrieved, 21 studies from 13 countries (Argentina, Australia, China, Egypt, Korea, India, Iran, Ireland, Israel, Italy, Morocco, Turkey, and the United Kingdom) were included (Figure 1. ); 15 reported clinical burden, 7 reported economic burden, and 4 reported humanistic burden 6 studies reported data on a combination of clinical, economic, and/or humanistic burden [Table 1]). Patients with delayed diagnosis of AxSpA generally had worse clinical outcomes, including higher disease activity (Bath Ankylosing Spondylitis Disease Activity Index), poorer mobility and physical function (Bath Ankylosing Spondylitis Functional Index), and more structural damage, compared with patients who had an earlier diagnosis (Table 1). Patients with delayed diagnosis also had higher healthcare costs, including costs of unnecessary treatments, and greater likelihood of work disability compared with those who had an earlier diagnosis (Table 1). Delayed diagnosis was associated with worse quality of life, including greater likelihood for depression and negative psychological impact (Table 1).

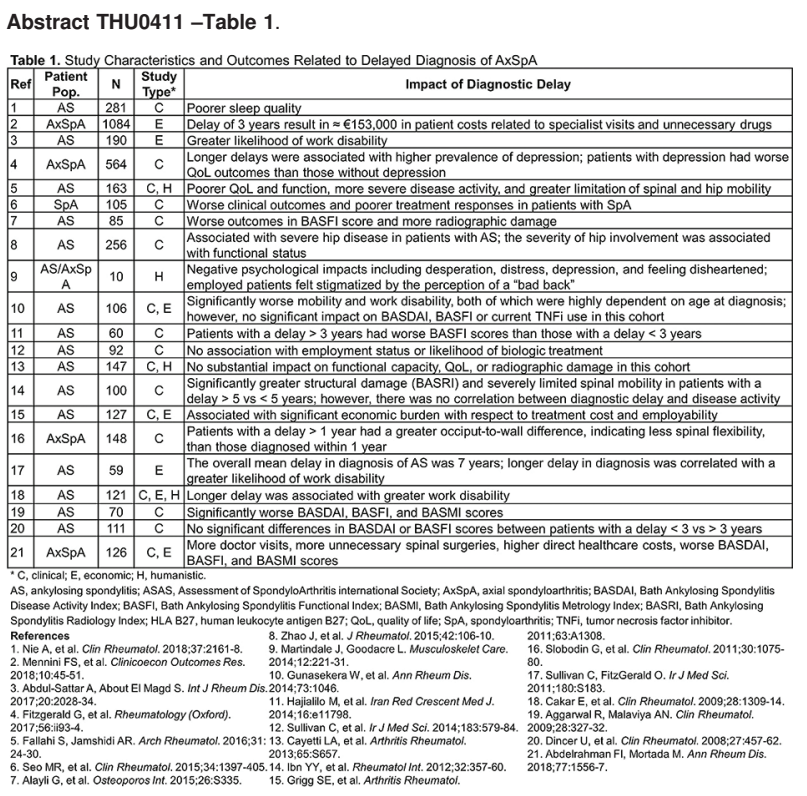

Conclusion: Delayed diagnosis in patients with AxSpA demonstrated a decrease in physical function, higher direct and indirect costs, and poorer quality of life. This study highlights the importance of early recognition and diagnosis of AxSpA in order to improve outcomes and mitigate extensive burden on patients and society. Therefore, further efforts by the healthcare community are warranted to increase awareness of early signs of disease and reduce the delay in diagnosis of AxSpA.

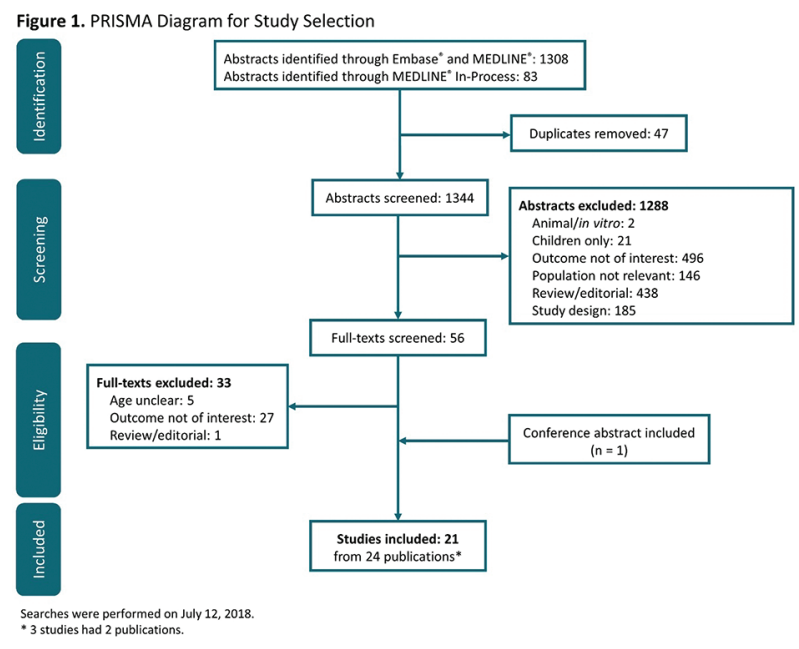

Abstract THU0411 - Figure 1

\section{REFERENCE:}

[1] Hutton B, et al. Ann Intern Med. 2015;163(7):566-7.

Acknowledgement: This study was sponsored by Novartis Pharmaceuticals Corporation, East Hanover, NJ.

Disclosure of Interests: Esther $\mathrm{Yi}$ Consultant for: $\mathrm{E}$. $\mathrm{Yi}$ is a postdoctoral fellow at the University of Texas at Austin and Baylor Scott and White Health, providing services to Novartis Pharmaceuticals Corporation., Amit Ahuja Employee of: A. Ahuja is an employee of Novartis Healthcare Pvt Ltd., Tanvi Rajput Employee of: T. Rajput is an employee of Novartis Healthcare Pvt Ltd., Aneesh George Employee of: Aneesh George is an employee of Novartis Healthcare Pvt Ltd., Yujin Park Employee of: Y. Park is an employee of Novartis.

DOI: 10.1136/annrheumdis-2019-eular.1003

\section{Osteoarthritis}

\section{THU0412 LONGITUDINAL ASSOCIATIONS BETWEEN MRI- DEFINED INFLAMMATION AND PAIN IN THUMB BASE OSTEOARTHRITIS}

Sjoerd van Beest ${ }^{1}$, H.M. Kroon ${ }^{2}$, Monique Reijnierse ${ }^{2}$, Frits Rosendaal ${ }^{3}$, Margreet Kloppenburg ${ }^{1,3}$, Féline P.B. Kroon ${ }^{1} .{ }^{1}$ Leiden University Medical Center, Rheumatology, Leiden, Netherlands; ${ }^{2}$ Leiden University Medical Center, Radiology, Leiden, Netherlands; ${ }^{3}$ Leiden University Medical Center, Clinical Epidemiology, Leiden, Netherlands

Background: : Hand osteoarthritis (OA) typically affects the interphalangeal (IP) joints and the thumb base (TB), including the first carpometacarpal (CMC-1) and scaphotrapeziotrapezoid (STT) joints. Based on previous studies, TB OA can be considered a distinct hand OA subset with a high burden of disease. In a cross-sectional study it was shown that TB pain is more strongly associated with radiographic damage than with MRI-defined inflammation, yet it is unknown if and how TB pain changes over time, and whether this is related to changes seen on imaging. Objectives: Our aim was to investigate the course of TB OA pain and its association with changes in MRI-defined inflammation and structural damage.

Methods: Longitudinal data of the Hand OSTeoArthritis in Secondary care (HOSTAS) study, which included patients diagnosed with primary hand $\mathrm{OA}$ by their treating rheumatologist, were used. Patients who underwent hand radiography, MR imaging and clinical examination of the right TB at baseline and two-year follow-up were studied. Pain on palpation of the TB was assessed by trained research nurses (0-3). Baseline and followup MR images were scored paired in known time-order by two readers following the OMERACT TB OA MRI scoring system (TOMS). The CMC- 